\title{
The Atmospheres of the Planets*
}

\section{By Dr. H. Spencer Jones, F.R.S., Astronomer Royal}

$\mathrm{D}^{\mathrm{L}}$ URING the last few decades, the main interests of astronomical research have shifted rapidly from the solar system outwards. Nevertheless, the planets have not been entirely neglected in recent research. The great lightgathering power of large modern telescopes has enabled spectrographs of very high dispersion to be used for the more detailed study of the spectra of the planets, and the great advances in the manufacture of plates sensitive to the infra-red region of the spectrum have made possible the investigation of a region of the spectrum the importance of which arises from the fact that the selective absorptions by planetary atmospheres lie mainly in this region.

From theoretical considerations it is possible to decide whether or not any planet may be expected to possess an atmosphere. The natural tendency of an atmosphere is to diffuse away into space. In order to overcome the gravitational pull of the planet any particle, whether large or small, must acquire a velocity greater than a certain minimum value, determined by the mass and radius of the planet.

The rate at which this loss of fast-moving molecules from the upper atmosphere takes place depends upon the relative magnitudes of the velocity of escape and of the mean velocity of the molecules. Jeans found that if the velocity of escape is four times the mean molecular velocity, the atmosphere would be practically completely lost in fifty thousand years; but if the velocity of escape is five times the mean molecular velocity, twenty-five thousand million years would be required for the loss to be almost complete. If therefore the velocity of escape is as great as five times the mean molecular velocity of hydrogen, the atmosphere will be practically immune from loss.

The velocities of escape from the moon and from Mercury are not much greater than the mean molecular velocity of hydrogen, so that an atmosphere of hydrogen would escape almost instantly from either body. It appears that the moon, if it had never been hotter than at present, would have lost water vapour, nitrogen and oxygen completely, but would have retained carbon dioxide and heavier gases; Mercury, under the same

- From the Norman Lockyer Lecture delivered under the auspices of the British Association on December 6. supposition, would have lost almost all its water vapour and nitrogen and most of its oxygen, but would have retained heavier gases to a large extent. The loss is likely to be under-estimated because, during the initial rapid cooling, the loss must have been considerable. It is certain that the moon has no atmosphere now. The evidence of an atmosphere on Mercury is not fully conclusive, but the opinion of Antoniadi that Mercury may possess a very tenuous atmosphere is not in conflict with the theoretical evidence.

\section{The EARTH}

Coming to the earth, the escape velocity is $11.2 \mathrm{~km}$./sec., which is almost exactly six times the mean molecular velocity of hydrogen at $0^{\circ} \mathrm{C}$. Hence the atmosphere of the earth should be immune from loss of hydrogen and all other gases. At the present time, the amounts of hydrogen and of helium in the earth's atmosphere are very small. The supply is being gradually replenished by the weathering of the igneous rocks of the earth's crust, which contain uranium and thorium and also consequently helium. Yet the atmosphere does not now contain more than a fraction of the amount of helium that it has gained in geological times. There is thus direct observational evidence that helium is being lost from the atmosphere at the present time.

There is one process by which the escape of helium can be brought about. It is well known that the night sky is faintly luminous. In the spectrum of the night sky the characteristic green auroral line, as well as the two red lines, are always present. These lines are emitted by oxygen atoms that are in what physicists term a metastable state. The atoms in a metastable state may remain for an average time of a second or longer in that state before emitting their energy in the form of radiation. There is a high probability that before this occurs the atom will have collided with another atom, when its energy will be converted into kinetic energy. By such a collision, an atom of hydrogen or of helium could acquire a speed greater than the velocity of escape from the earth, but an atom of nitrogen or oxygen would not by this process acquire sufficient speed to escape. The loss of hydrogen and helium from 
the atmosphere of the earth is thus made possible by the fact that free oxygen is present in the atmosphere.

It appears probable that the primitive earth must have remained hot sufficiently long for most of its initial atmosphere to have been lost. It was pointed out by Russell and Menzel that in the stars and the nebulæ neon is as abundant as argon, whereas in the earth's atmosphere argon is five hundred times more abundant than neon. Nitrogen is far less abundant on the earth than in the stars. These large differences in relative terrestrial and solar abundance can be accounted for on the supposition that the rate of loss of atmosphere was very rapid when the earth was hot. When the cooling had proceeded sufficiently far for the escape of the atmosphere to cease, neon had been depleted to a much greater extent than the heavier argon. If this supposition is correct, much of the original oxygen, nitrogen and water vapour and all the original helium and free hydrogen must have been lost. As the molten earth cooled, great quantities of water vapour, carbon dioxide and other gases would have been evolved from the solidifying magma; these, with the residual gases from the initial atmosphere, formed the new atmosphere which, as the earth was then relatively cool, could not escape.

The presence of free oxygen in the atmosphere of the earth needs explanation, for processes are in continual operation that are depleting the store. One of the principal causes of depletion is the weathering of the igneous rocks to form sedimentary deposits which is occurring. In this process much of the ferrous oxide is oxidized to ferric oxide. It has been estimated that during geological times the amount of oxygen thus depleted from the atmosphere is about twice the quantity now present. It is clear that the oxygen must be replenished in some way, and this is done by green vegetation on the earth's surface.

\section{VENUS}

The atmosphere of Venus is in marked contrast to that of the earth. The velocity of escape from Venus is $10.2 \mathrm{~km}$./sec. It may therefore be expected that Venus will have an atmosphere comparable with that of the earth in extent and density. The presence of an extensive atmosphere is confirmed by observation. Photographs in ultra-violet light record cloudy markings which rapidly change their form and are of short duration.

To determine the composition of the atmosphere of Venus, or of any other planet, recourse must be had to the spectroscope. Absorption in the atmosphere of the earth is a complicating and troublesome factor. These absorptions can be identified in two ways. First, by observing the spectrum of the sun at different altitudes; the terrestrial absorptions become stronger the lower the altitude. Secondly, if the spectra of light from the east and west limbs of the sun are compared, the absorptions of solar origin show a slight relative displacement caused by the solar rotation, whilst the absorptions of terrestrial origin are undisplaced. The absorptions of terrestrial origin having been identified, the absorptions produced in the atmosphere of a planet can be investigated by photographing the spectra of the planet and the moon on the same night and at the same altitude. Any absorption present in the spectrum of the planet and not in that of the moon, or much stronger in the spectrum of the planet than in that of the moon, must originate in the atmosphere of the planet. Another, and more delicate, method of investigation is to photograph the spectrum of the planet at a time when it is approaching or receding from the earth most rapidly. The absorptions due to the planet's atmosphere are displaced with respect to those due to our own atmosphere.

Complete information about the constitution of any planetary atmosphere is not obtainable, however, because many possible constituents of the atmosphere show no absorptions in the region accessible to study. Amongst such undetectable constituents are hydrogen, nitrogen, helium, neon and argon.

The investigation of the atmosphere of Venus has given no certain evidence of the presence of oxygen. Observations have led to the conclusion that the amount of oxygen must be less than one thousandth part of that above an equal area of the earth. More surprising, perhaps, than the failure to detect oxygen, is the failure to detect the presence of water vapour, even though the tests for water vapour are less sensitive than those for oxygen.

The most interesting fact about the atmosphere of Venus is the great abundance of carbon dioxide. In 1932, Adams and Dunham discovered three welldefined bands in the infra-red region of the spectrum of Venus. These bands had not at that time been observed in any terrestrial spectrum. Theoretical investigations indicated that they might be due to carbon dioxide; this was first confirmed by Dunham. Later, Adel and Slipher reproduced the three bands by passing light through 45 metres of carbon dioxide at a pressure of 47 atmospheres; the absorptions so obtained were less intense than the corresponding absorptions in the spectrum of Venus. They concluded that the amount of carbon dioxide above the surface of 
Venus is equivalent to a layer two miles in thickness at standard atmospheric pressure and temperature. For comparison, it may be mentioned that the amount of carbon dioxide present in the earth's atmosphere is equivalent to a thickness of only about 30 feet.

The carbon dioxide will have a powerful blanketing effect, and it is not improbable that the temperature at the surface of Venus may be as high as, or higher than, that of boiling water. The high temperature, the lack of oxygen and the abundance of carbon dioxide can be interpreted as indications that there cannot be any great amount of vegetation on Venus.

\section{MARS}

Mars occupies a position between Mercury on one hand and Venus and the earth on the other, as regards size, mass and velocity of escape. The velocity of escape is $5.0 \mathrm{~km}$./sec. It may be expected that Mars will have a much thinner atmosphere than Venus or the earth. The presence of an atmosphere on Mars can be proved by photographing the planet in light of different colours. Photographs in the infra-red show permanent markings, which are evidently surface features, whereas photographs in the ultra-violet show none of these. The atmosphere is extensive enough to scatter ultra-violet light to such an extent that the light cannot penetrate to the surface and out again. The difference in size of the images in ultra-violet and infra-red light indicates that the atmosphere extends to a height of fully fifty miles above the surface.

The polar caps provide additional evidence of an atmosphere on Mars. They wax and wane with the changing of the seasons The changes are to be explained by the melting or deposition of ice, snow or hoar-frost, and, from the rate at which the caps decrease as summer advances it can be calculated that they are not more than a few inches thick.

All attempts to detect oxygen in the atmosphere of Mars have been unsuccessful. The amount of oxygen is certainly not more than one thousandth part of the amount in the earth's atmosphere. The red colour of Mars, which is unique among the heavenly bodies, provides indirect evidence of oxygen, suggesting rocks that have been completely oxidized. The amount of water vapour in the atmosphere of Mars is so small that it can be detected only under the most favourable conditions. At the Lowell Observatory, which is at an altitude of 7,250 feet, Slipher, in 1908, by comparing the spectra of Mars and the moon when at the same altitude under conditions of exceptional atmospheric dryness, found that the water vapour absorptions were slightly stronger in the spectrum of Mars than in that of the moon.

Carbon dioxide has not been detected in the Martian atmosphere. There is some evidence of the existence of vegetation on Mars. Seasonal changes in form and coloration of the dark areas seem to be reasonably well established, and the interpretation of these changes as due to the seasonal growth of vegetation is plausible. The presence of some carbon dioxide in the atmosphere may therefore be inferred. Mars appears to be a world in the state that the earth will ultimately reach when the oxygen in the atmosphere will have been almost entirely exhausted by the progressive weathering and oxidation of the rocks.

\section{The Major Planets}

The major planets, Jupiter, Saturn, Uranus and Neptune, may be considered together. They are large massive planets, of low mean density, the visible disks of which are considerably oblate. Saturn has the lowest mean density of any of the planets, only seven tenths that of water. The velocities of escape from all the major planets are so high that extensive atmospheres are to be expected, containing an abundance of hydrogen and helium.

The telescopic appearance of Jupiter and Saturn confirms the existence of dense atmospheres. Markings in the form of belts parallel to the equator may be seen; these are of complex structure and their details are continually changing. Photographs in the infra-red show many differences from those in the ultra-violet, but again the recorded features are continually changing, so that the infra-red light does not penetrate to the surface.

Some theoretical results of interest have been deduced from the oblateness and mean density of these planets. Jeffreys has concluded that they consist of a core of rock, generally similar to the inner planets in its constitution and of about the same mean density, surrounded by ice-coatings of great depth, above which are very extensive atmospheres. According to the calculations by Wildt, the rocky core of Jupiter has a radius of about 22,000 miles: the ice-coating is 16,000 miles in thickness and the depth of the atmosphere is about 6,000 miles. The rocky core of Saturn is about 14,000 miles in radius ; it is covered with a layer of ice some 6,000 miles thick, over which is an atmosphere extending to a height of 16,000 miles. Saturn has the most extensive atmosphere of any of the planets, which explains why it has the lowest mean density and 
the most flattened disk. The pressures of these extensive atmospheres are very great; at the bottom of Jupiter's atmosphere, for example, the pressure is fully a million times the pressure at the bottom of the earth's atmosphere. At a relatively small depth in the atmosphere, the pressure is great enough to compress the gas to a density nearly equal to that of the corresponding liquid.

The densities of the atmospheres are low; according to Wildt's calculations, they are 0.78 for Jupiter and 0.41 for Saturn. This enables most of the possible constituents to be excluded, for all known gases, in the liquid or solid state, have densities exceeding $0 \cdot 3$, with the exceptions of hydrogen and helium, and the only other gases whose densities in the liquid or solid state are less than the density of the greater portion of the atmosphere of Jupiter are methane and ethane. It is concluded that the atmospheres of the major planets must contain large quantities of free hydrogen and helium. The planets are believed to have been formed from the sun, which is known to contain a large amount of hydrogen, to the extent of about one third part by weight. As massive planets, like the four major planets, would retain their light constituents, it is to be expected that hydrogen and helium would be present in large amount in their atmospheres.

\section{Planetary Spectra}

The spectra of the major planets are of great interest. Huggins discovered visually absorption bands in the orange and green in the spectrum of Jupiter. These bands appear more strongly in the spectrum of Saturn, but are not found in the spectrum of the rings-a conclusive proof that they originate in the atmosphere of Saturn. Uranus and Neptune show for the most part the same bands with still greater intensity, together with some additional ones. In recent years, the spectra have been extended far into the infra-red by Slipher, and several intense bands have been found in that region.

The origin of these bands remained unknown until a few years ago. Then Wildt succeeded in proving theoretically that certain of the bands agreed in position with bands of ammonia and that others agreed with bands of methane. These conclusions were confirmed by Dunham who, using much higher dispersion, was able to obtain a more complete resolution of the bands into their component lines and found a complete coincidence. He estimated that the quantity of ammonia gas producing the absorptions in the spectrum of Jupiter is equivalent to a layer 30 feet thick under standard conditions. The amount is less in Saturn. The ammonia absorptions are not detected in the spectra of Uranus and Neptune.

Methane is present in much larger amount. Adel and Slipher found that a 45-metre path of methane, at a pressure of 40 atmospheres, gave bands intermediate in intensity between those of Jupiter and Saturn. The much greater strength of the methane absorptions in Uranus and Neptune is probably accounted for by the lower temperatures of these planets. The ammonia must be frozen out of their atmospheres, making it possible to see through them to a greater depth. Adel and Slipher estimated that 25 miles of methane at atmospheric pressure would be required to give absorptions as strong as those of Neptune. The higher gaseous hydrocarbons have been looked for in vain in the spectra of the outer planets. All the absorption bands appear to be accounted for by ammonia and methane. It is a grand slam.

The presence of ammonia and methane in the atmospheres of the large planets is not surprising. They are to be expected as a consequence of the reaction of hydrogen with nitrogen and carbon dioxide on a cooling planet. Such reactions resulted in an atmosphere of hydrogen, helium, and other inert gases, mixed with methane, ammonia and water vapour, but with little or no carbon dioxide or free nitrogen. Below this there would be a deep ocean, strongly alkaline from the ammonia in solution. As the temperature fell still further, the ocean would freeze. It may be mentioned that an ocean consisting of one part of ammonia to two parts of water would freeze at $-100^{\circ} \mathrm{C}$; ; all the four major planets are colder than this.

The ammonia in the atmospheres of Jupiter and Saturn must be nearly on the point of condensation, and the clouds over these planets may consist of droplets of liquid ammonia or even small crystals of frozen ammonia. The mean temperatures of Uranus and Neptune due to solar radiation alone are about $-200^{\circ} \mathrm{C}$. and $-220^{\circ} \mathrm{C}$. respectively. At the temperature of Neptune, the methane must be nearly ready to condense.

The nature of the planetary atmospheres, about which so little was known until recently, seems now to have been solved in its broad outlines. As a brief summary we find that we can divide the planets and their satellites into three groups: the small ones, entirely devoid of atmospheres; the middle-sized ones with atmospheres of moderate extent, devoid of hydrogen or compounds of hydrogen with nitrogen or carbon but containing oxygen or compounds of oxygen; and the large ones, with very extensive atmospheres, devoid of oxygen or compounds of oxygen but containing hydrogen and compounds of hydrogen. 